\title{
The Circadian Clock Regulates the Expression of the Nuclear Factor Erythroid 2-Related Factor 2 in Acute Kidney Injury following Myocardial Ischemia-Reperfusion in Diabetic Rat
}

\author{
Chong Dong $\mathbb{D},{ }^{1,2}$ Cheng Zeng $\mathbb{D}^{3},{ }^{3}$ Li Du $\mathbb{D}^{3},{ }^{3}$ and Qian Sun $\mathbb{D}^{3}$ \\ ${ }^{1}$ Organ Transplantation Center, Tianjin First Central Hospital, Tianjin 300192, China \\ ${ }^{2}$ Tianjin Key Laboratory for Organ Transplantation, Tianjin 300192, China \\ ${ }^{3}$ Department of Anesthesiology, Renmin Hospital of Wuhan University, Wuhan 430060, China \\ Correspondence should be addressed to Qian Sun; queenie_sun@whu.edu.cn
}

Received 18 November 2020; Revised 14 January 2021; Accepted 3 February 2021; Published 25 February 2021

Academic Editor: Bing Wang

Copyright (C) 2021 Chong Dong et al. This is an open access article distributed under the Creative Commons Attribution License, which permits unrestricted use, distribution, and reproduction in any medium, provided the original work is properly cited.

Cardiac surgery-associated acute kidney injury (AKI) is a serious and frequent complication with poor prognosis, and disruption in circadian rhythm shall adversely influence cardiovascular and renal functions via oxidative stress mechanisms. However, the role of circadian clock genes (circadian locomotor output cycle kaput (CLOCK) and brain and muscle aryl hydrocarbon receptor nuclear translocator-like protein-1 (BMAL1)) and its interaction with nuclear factor erythroid 2-related factor 2 (Nrf2) in AKI following myocardial ischemia-reperfusion (MIR) in the diabetic rat has not yet been explored. In this study, rats were divided into the sham (S) group, MIR (M) group, diabetic (D) group, and diabetic+MIR (DM) group. At light (zeitgeber time (ZT) 0) and dark time points (ZT12), rat MIR model was established by occlusion of the left anterior descending coronary artery for 30 min followed by 2 -hour reperfusion, and then renal injury was evaluated. The renal histological changes in the DM group were significantly high compared to other groups; serum creatinine, blood urea nitrogen, and neutrophil gelatinase-associated lipocalin levels, as well as malondialdehyde and 8-iso-prostaglandin-F2 $\alpha$ levels in renal tissues of M ZT12 and DM ZT12 subgroups, were significantly higher than those of M ZT0 and DM ZT0 subgroups, individually indicating increased oxidative stress at a dark cycle. Further, Nrf2 protein accumulated in a circadian manner with decreasing levels at night in the DM and M groups. In conclusion, renal injury following MIR was exacerbated in the diabetic rat at night through molecular mechanisms involving transcriptional control of the circadian clock on light-dark activation of Nrf2.

\section{Introduction}

Cardiac surgery-associated acute kidney injury (AKI) is a serious complication with high morbidity and increased cardiac surgery mortality [1]. Diabetes is an independent risk factor of AKI, as well as the most common complication in patients undergoing cardiac surgery [2]. Interestingly, clinical studies have shown that the incidence of adverse events after on-pump cardiac surgeries performed in the afternoon is significantly higher than the cardiac surgeries performed during the daytime. Thus, it is increasingly clear that circadian clocks may likely mediate some of these cardiovascular observations [3]. However, whether AKI following myocardial ischemia-reperfusion (MIR) in the diabetic rat is associ- ated with the circadian cycle of 12-hour (h) light/12-hour dark (LD) has not yet been investigated.

The suprachiasmatic nucleus (SCN) of the brain plays a primary role in synchronizing the peripheral organs' circadian clock rhythms to the LD cycle [4]. However, the circadian cycle is sensitive to many other factors, as numerous diseases can disrupt circadian rhythms [5]. Specifically, the circadian disruption is associated with the pathophysiology of diabetes [6], and changes in the circadian regulation of renal function influence diurnal blood pressure and urinary sodium excretion [7], which may lead to some circadian physiological problems including nondipping blood pressure, diurnal urinary sodium excretion disturbance, sleepwake disturbance, and restless leg syndrome in patients with 
chronic kidney disease [8]. However, the circadian clock gene's involvement in AKI following MIR in diabetic rats has not been studied yet.

Our previous study results have indicated that oxidative stress plays a chief role in AKI following MIR in diabetic rats $[9,10]$. Lately, an increasing number of researchers explored the contribution of circadian clock gene regulation in the antioxidative stress mechanisms, especially through the nuclear factor erythroid 2-related factor 2 (Nrf2)/antioxidant response element (ARE) pathway $[11,12]$. Recent studies have shown that the circadian transcription factors such as circadian locomotor output cycle kaput (CLOCK) and brain and muscle aryl hydrocarbon receptor nuclear translocator(ARNT-) like protein 1 (BMAL1) can regulate the expression of Nrf2 and its downstream antioxidant stress proteins binding to peroxisome proliferator-activated receptor (Ppar) promoter through an E-Box element, which mimics the involvement of the transcription of ARE and other key antioxidant proteins in circadian rhythm $[13,14]$. However, the mechanistic pathway involving the circadian clock that regulates the expression of $\mathrm{Nrf} 2$, as a newly discovered key protein and regulator of oxidative stress, to activate the downstream antioxidant protein and regulate the antioxidant capacity of AKI following MIR in the diabetic rat is not yet established. Hence, in this study, we aimed to explore the pivotal role of Nrf2/ARE pathway controlled by the circadian clock that exerts protection against oxidative stress injury in AKI following MIR in diabetic rats.

\section{Materials and Methods}

2.1. Materials. The experimental protocols used in this study were approved by the Laboratory Animal Welfare \& Ethics Committee (IACUC) of Wuhan University (No. 2020-0323), and the animal experiments were conducted in accordance with the guidelines for the Care and Use of Laboratory Animals by the National Institutes of Health (NIH Publications No. 8023, revised 1978). Adult male Sprague-Dawley (SD) rats (weighing 220-250 g, 6-8 weeks old) (Wuhan University, Wuhan, China) were housed in a specific pathogenfree animal facility. At least 10 days before experimentation, the rats were acclimatized in a $12 \mathrm{~h}$ light $/ 12 \mathrm{~h}$ dark cycle (lights on at zeitgeber time (ZT) 0). The SD rats received standard laboratory chow and water. Streptozotocin (STZ) was purchased from Sigma-Aldrich Co. (St. Louis, MO, USA). Antibodies for assessing circadian clock proteins BMAL1, CLOCK, period 2 (PER2), and Nrf2 were purchased from Novus Biologicals (Littleton, CO, USA) and Abcam plc. (Cambridge, UK). $\beta$-Actin and secondary antibodies against Lamin B were purchased from Cell Signaling Technology, Inc. (Beverly, MA, USA), and horseradish peroxidase(HRP-) conjugated secondary antibodies were purchased from Santa Cruz Biotechnology, Inc. (Santa Cruz, CA, USA). Blood urea nitrogen (BUN) and serum creatinine (Scr) were measured using an Olympus automatic analyzer, and 8-iso-prostaglandin-F2 $\alpha$ (8-iso-PGF2 $\alpha$ ) and neutrophil gelatinase-associated lipocalin (NGAL) levels were quantified using the corresponding enzyme-linked immunosorbent assay (ELISA) kits (Elabscience Biotechnology Co. Ltd.,
Wuhan, China). Superoxide dismutase (SOD) and malondialdehyde (MDA) assay kits were purchased from Nanjing Jiancheng Biochemicals Ltd. (Nanjing, China).

2.2. Induction of Diabetes. Following acclimatization, experimental diabetes in the SD rats was induced by a single intraperitoneal injection of STZ solution in citrate buffer at $65 \mathrm{mg} / \mathrm{kg}$ [15], while a single intraperitoneal injection of citrate buffer alone served as a control. Three days post-STZ injection, the tail vein blood glucose was measured using the OneTouch glucometer (Johnson \& Johnson, NJ, USA), and rats with blood glucose levels $>15 \mathrm{mmol} / \mathrm{L}$ were regarded as successful diabetic models. Rats were housed 8 weeks after vehicle or STZ injection $[15,16]$.

2.3. Surgical Preparation and MIR Model [15]. All SD rats were anesthetized (intraperitoneal injection of $2 \%$ pentobarbital sodium $40 \mathrm{mg} / \mathrm{kg}$ ), intubated, and mechanically ventilated $(12 \mathrm{~mL} /$ per, 70 per $\mathrm{min}$, No. ALC-V9, SHANGHAI ALCOTT BIOTECH CO., China). Blood pressure was recorded from the left femoral artery using a pressure transducer with heart rate monitored by an electrocardiogram (ECG) throughout the procedure. The MIR model was established by the left anterior descending coronary artery (LAD) occlusion for ischemia $(30 \mathrm{~min})$ and then removing the microvascular clip for $2 \mathrm{~h}$ reperfusion [10]. After $2 \mathrm{~h}$ reperfusion, cardiac and renal tissues and blood samples were obtained for further evaluation.

2.4. Animal Experimental Protocols. The SD rats were randomly divided into 4 groups ( $n=8$ per group) as follows: (1) in the sham (S) group, except occlusion, the other above-mentioned surgical procedures were performed; (2) in the MIR (M) group, the MIR model was established by LAD occlusion followed by reperfusion in normal rats; (3) in the diabetic (D) group, diabetic rats underwent isolation of the LAD without occlusion; and (4) in the diabetic+MIR (DM) group, the MIR model was established in diabetic rats. The LAD was occluded for $30 \mathrm{~min}$ by tightening the ligature at ZT0 and ZT12 individually.

2.5. Myocardial and Renal Histopathological Assessment. The tissues from the heart apical region and left kidney were sectioned and fixed with $4 \%$ formaldehyde for $24 \mathrm{~h}$. After paraffin embedding, $4 \mu \mathrm{m}$ sections were stained with hematoxylin for $3 \mathrm{~min}$ at room temperature and eosin for $60 \mathrm{sec}$. The stained samples were evaluated using the Olympus BX50 fluorescence microscope (Original magnification $\times 200$ ), and the renal histological grading was performed using the semiquantitative scale described previously by Spandou et al. [17]. For each kidney, at least 100 cortical tubules from 10 different regions were scored. Higher scores represented more severe damage (maximum score per tubule was 10), with points as follows: $0=$ normal kidney; $1=$ minimal damage $(<5 \%$ involvement of the cortex or outer medulla); $2=$ mild damage (5-25\% involvement of the cortex or outer medulla); $3=$ moderate damage $(25-75 \%$ involvement of the cortex or outer medulla); and $4=$ severe damage ( $>75 \%$ involvement of the cortex or outer medulla). 

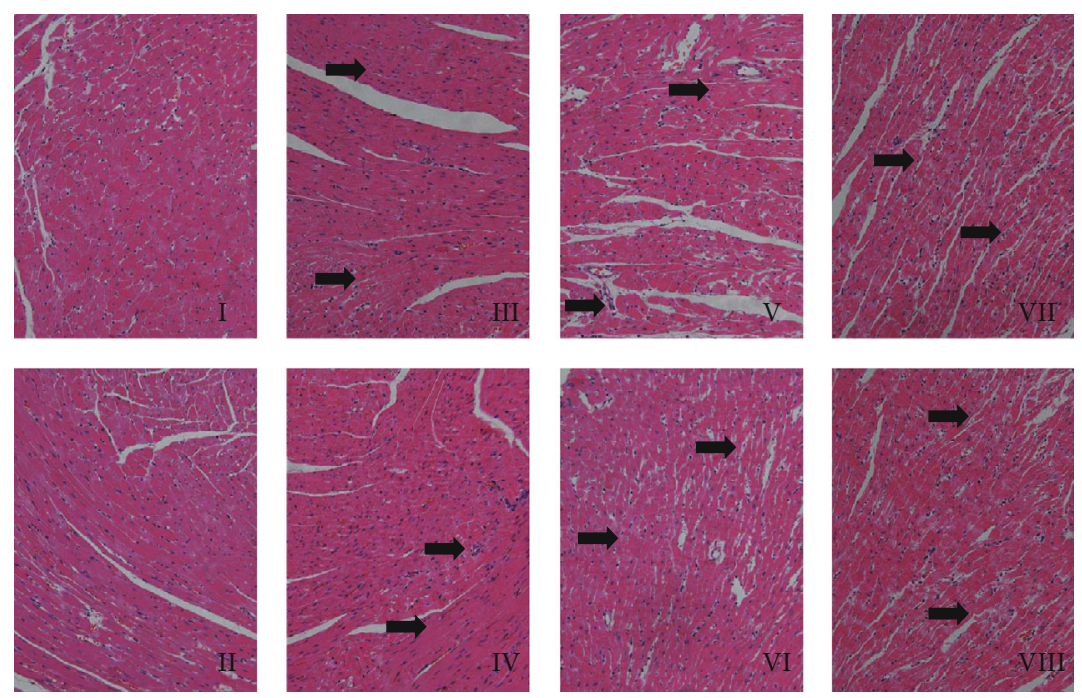

I: S ZT0

II: S ZT12

III: D ZT0

IV: D ZT12
V: M ZT0

VI: M ZT12

VII: DM ZT0

VIII: DM ZT12

(a)

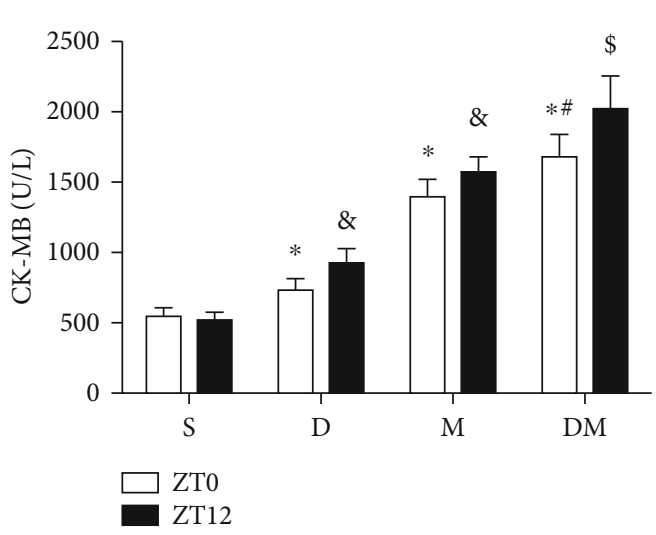

(b)

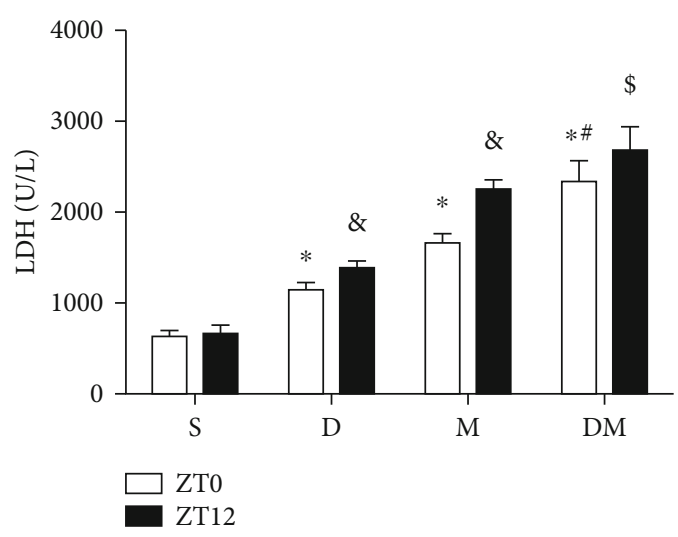

(c)

FIGURE 1: Myocardial injury assessment. (a) Pathological changes in the heart (HE $\times 200)$. I: S ZT0 group; II: S ZT12 group; III: D ZT0 group; IV: D ZT12 group; V: M ZT0 group; VI: M ZT12 group; VII: DM ZT0 group; VII: DM ZT12 group. (b, c) CK-MB and LDH levels in serum. $n=8$ per group; ${ }^{*} P<0.05$ compared to the values of the $S$ group; ${ }^{\#} P<0.05$ compared to the values of $\mathrm{M} Z \mathrm{ZT} 0$ group; ${ }^{\$} P$ and ${ }^{\&} P<0.05$ compared to the values of M ZT0 and DM ZT0 groups, respectively.

2.6. Measurement of Serum Creatine Kinase Isoenzyme $M B$ (CK-MB) and Lactate Dehydrogenase (LDH) Levels. Blood samples were collected at the end of reperfusion and centrifuged at $3000 \mathrm{rpm}$, for $10 \mathrm{~min}$ at $4^{\circ} \mathrm{C}$. Serum was separated and stored at $-20^{\circ} \mathrm{C}$. CK-MB and LDH levels were measured using commercial kits (Beijing Kemeidongya Biotechnology Ltd., China) according to the manufacturer's instructions.

2.7. Measurement of Serum Scr, BUN, and NGAL. At the end of the MIR, the rats' right internal carotid artery was isolated, and $2 \mathrm{~mL}$ of blood was collected for each group. Blood samples were centrifuged at $3000 \mathrm{~g}$ for $10 \mathrm{~min}$, and then serum was separated at $4^{\circ} \mathrm{C}$ and stored at $-20^{\circ} \mathrm{C}$. Scr and BUN were measured using an Olympus automatic analyzer (AU5400; Olympus Corporation), and NGAL levels were measured using the corresponding ELISA assay kits.
2.8. Measurement of SOD, MDA, and 8-iso-PGF2 $\alpha$ Levels in Renal Tissues. Renal tissues were homogenized with normal saline on ice and centrifuged at $1200 \mathrm{~g}$ for $10 \mathrm{~min}$. The SOD activity and MDA levels were determined using a chemical assay kit following the manufacturer's instructions. The 8 -iso-PGF2 $\alpha$ levels were quantified using the ELISA assay kit.

2.9. Western Blot Analysis. Cytoplasmic and nuclear proteins of renal tissues were extracted using commercial protein extraction kits (Beyotime Institute of Biotechnology, Haimen, China), according to the manufacturer's instructions. After measuring the protein concentration, an equal amount of protein was loaded onto $12 \%$ sodium dodecyl sulfate-polyacrylamide gel electrophoresis and then transferred to membranes. Subsequently, the membranes were 

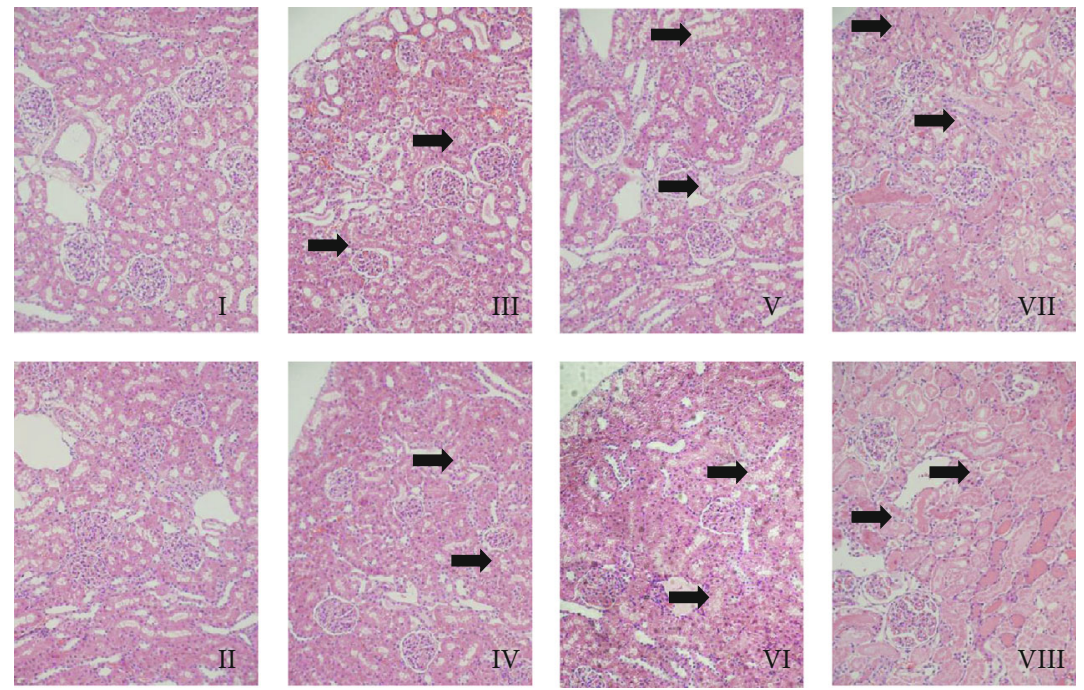

$$
\begin{aligned}
& \text { I: S ZT0 } \\
& \text { II: S ZT12 } \\
& \text { III: D ZT0 } \\
& \text { IV: D ZT12 }
\end{aligned}
$$

V: M ZT0

VI: M ZT12

VII: DM ZT0

VIII: DM ZT12

(a)

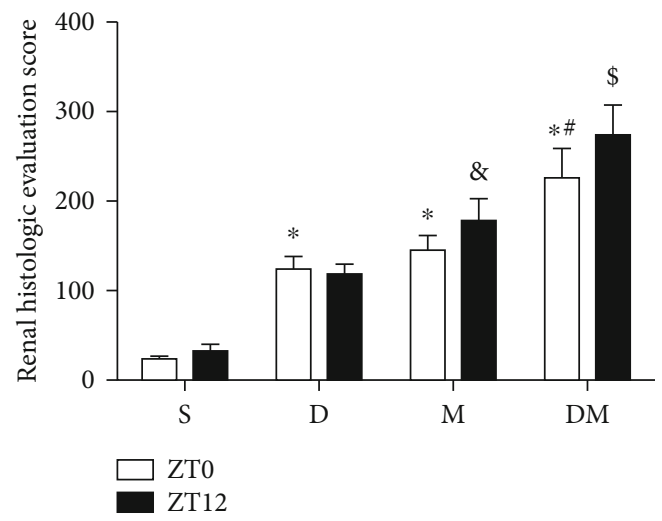

(b)

FIGURe 2: Renal histopathological assessment. (a) Pathological changes in the kidney (HE $\times 200)$. I: S ZT0 group; II: S ZT12 group; III: D ZT0 group; IV: D ZT12 group; V: M ZT0 group; VI: M ZT12 group; VII: DM ZT0 group; VII: DM ZT12 group. (b) Histologic evaluation score in the kidney. $n=8$ per group; ${ }^{*} P<0.05$ compared to the values of the $S$ group; ${ }^{\#} P<0.05$ compared to the values of the M ZT0 group; ${ }^{\$} P$ and ${ }^{\&} P<0.05$ compared to the values of the M ZT0 and DM ZT0 groups, respectively.

blocked with $5 \%$ nonfat milk and incubated overnight at $4^{\circ} \mathrm{C}$ with specific primary antibodies for CLOCK, BMAL1, PER2, and Nrf2 (each at 1:800 dilution). After washing with Tris-buffered saline plus $0.1 \%$ Tween 20 repeatedly, the membranes were incubated with the fluorescent secondary antibody $(1: 10000)$ for $1 \mathrm{~h}$ at room temperature. The immunoreactive bands were visualized by enhanced chemiluminescence and captured on X-ray films. The optical density of the bands was measured with Glyko ${ }^{\circledR}$ BandScan V4.0 imaging analysis system.

2.10. Statistical Analysis. All data were expressed as the mean \pm standard deviation values and were analyzed using GraphPad Prism 8.0 (GraphPad Software Inc., La Jolla, CA, USA). Statistical significance of differences among groups was determined using a two-way analysis of variance with
Tukey's post hoc test. A comparative analysis between the 2 groups was performed using a $t$-test. A $P$ value of less than 0.05 was considered statistically significant.

\section{Results}

3.1. Assessment of Myocardial Injury. Myocardial histopathological injury demonstrated the disorder and necrosis of cardiomyocytes and neutrophil infiltration in $M$ groups, and the histological changes in the DM groups were found to be significantly outstanding in Figure 1(a).

Serum CK-MB and LDH levels as indicators of myocardial cellular injury were measured at the end of reperfusion in the different groups. Compared with the S group, MIR caused a significant increase in $\mathrm{LDH}$ and CK-MB in both 


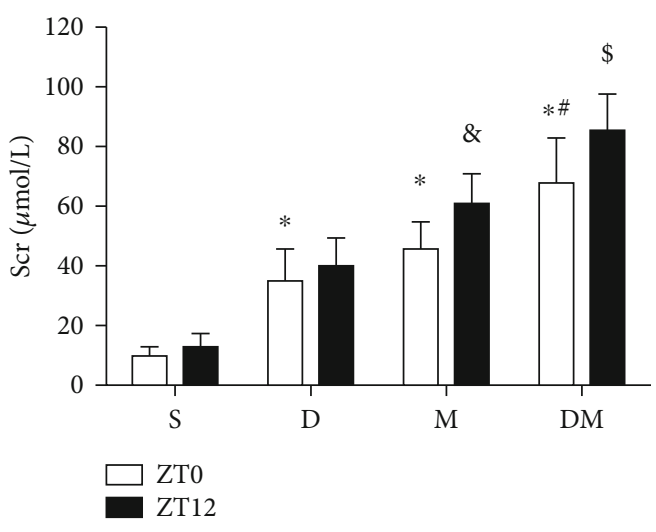

(a)

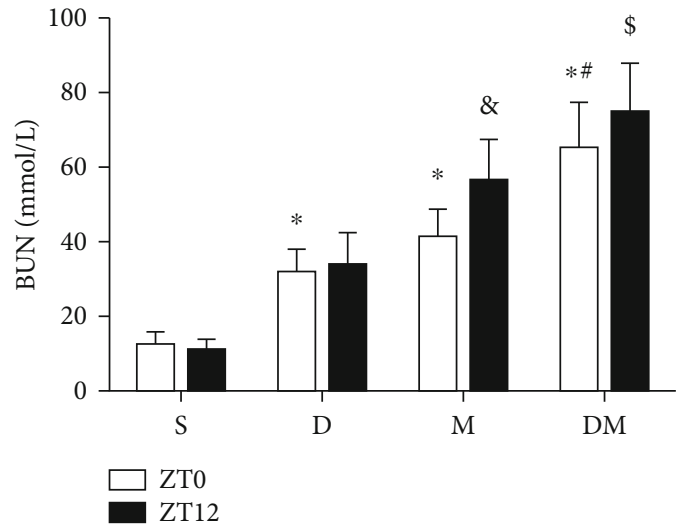

(b)

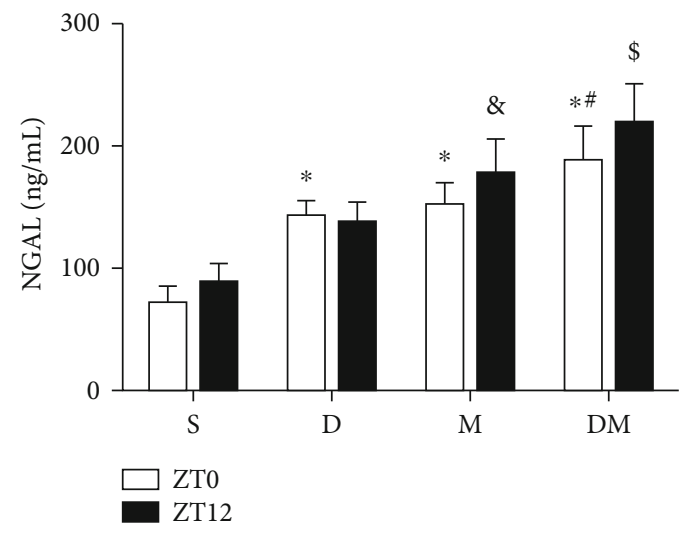

(c)

Figure 3: Scr (a), BUN (b), and NGAL (c) levels in serum. $n=8$ per group; ${ }^{*} P<0.05$ compared to the values of $S$ group. ${ }^{\#} P<0.05$ compared to the values of the M ZT0 group; ${ }^{\$} P$ and ${ }^{8} P<0.05$ compared to the values of the M ZT0 and DM ZT0 groups, respectively.

ZT0 and ZT12 of the M groups that was further enhanced in the DM groups $(P<0.05$, Figures $1(\mathrm{~b})$ and $1(\mathrm{c}))$.

3.2. Renal Histopathological Assessment. As shown in Figure 2(a), the main renal histopathological injuries were swelling, vacuolization, and necrosis of renal tubular epithelial cells in the $\mathrm{M}$ and $\mathrm{D}$ groups, and compared to those of other groups, the renal histological changes in the DM group were found to be significantly high. As shown in Figure 2(b), compared with normal kidney, the renal histological grading scores in the $\mathrm{M}$ and $\mathrm{D}$ groups were significantly increased $(P<0.05)$. This increase was significantly high in the DM group than in the $\mathrm{M}$ group $(P<0.05)$. Furthermore, the renal histological grading scores of the M ZT12 and DM ZT12 subgroups were significantly higher than those of the M ZT0 and DM ZT0 subgroups, individually $(P<0.05)$.

3.3. Serum Scr, BUN, and NGAL Levels. The Scr ( $\mu \mathrm{mol} / \mathrm{L})$, BUN (mmol/L), and NGAL $(\mathrm{ng} / \mathrm{mL})$ levels were determined to evaluate the AKI following MIR in diabetic rats. As shown in Figure 3, the Scr, BUN, and NGAL levels were significantly increased in the $\mathrm{M}$ and $\mathrm{D}$ groups compared to the $\mathrm{S}$ group $(P<0.05)$. This increase was significantly high in the DM group than in the $\mathrm{M}$ group $(P<0.05)$. Furthermore, the Scr, BUN, and NGAL levels of the M ZT12 and DM ZT12 subgroups were significantly higher than those of the $M$ ZT0 and DM ZT0 subgroups, individually $(P<0.05)$.

3.4. SOD Activity, MDA, and 8-Iso-PGF $2 \alpha$ Levels in Renal Tissues. As shown in Figure 4, the MDA ( $\mathrm{nmol} / \mathrm{mg}$ protein) and 8 -iso-PGF $2 \alpha$ (pg/mg protein) levels were significantly increased, while the SOD activity (U/mg protein) had decreased in the $\mathrm{M}$ and $\mathrm{D}$ groups compared with the $\mathrm{S}$ group $(P<0.05)$. As the indicators of oxidative stress, this increase in MDA and 8-iso-PGF $2 \alpha$ levels and a decrease in SOD activity were aggrandized in the DM group than in the $\mathrm{M}$ group $(P<0.05)$. Furthermore, the MDA and 8 -iso-PGF2 $\alpha$ levels of the M ZT12 and DM ZT12 subgroups were significantly higher than those of the M ZT0 and DM ZT0 subgroups individually, and similarly, the SOD activity was significantly low in the M ZT12 and DM ZT12 subgroups compared to the M ZT0 and DM ZT0 subgroups individually $(P<0.05)$.

\subsection{Renal Tissues CLOCK, BMAL1, PER2, and Nrf2 Protein} Expressions by Western Blot. Given their known functions, we hypothesized that the induction of circadian clock genes, especially BMAL1, could result in the activation of Nrf2 gene oscillations followed by diabetes and IR injury. Hence, the effects of diabetes and MIR on the circadian clock and Nrf2 protein expressions in renal tissues were investigated in this study. As shown in Figure 5, at ZT0, CLOCK and BMAL1 


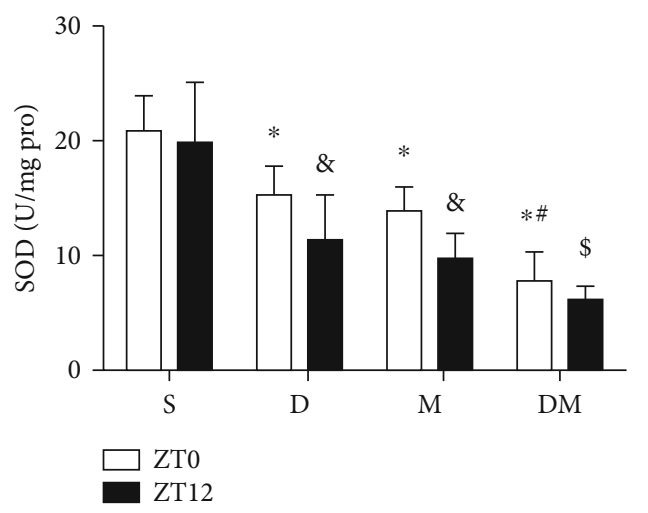

(a)

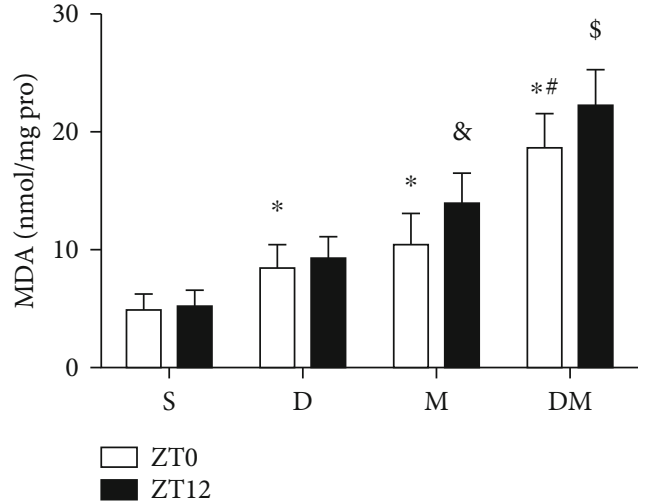

(b)

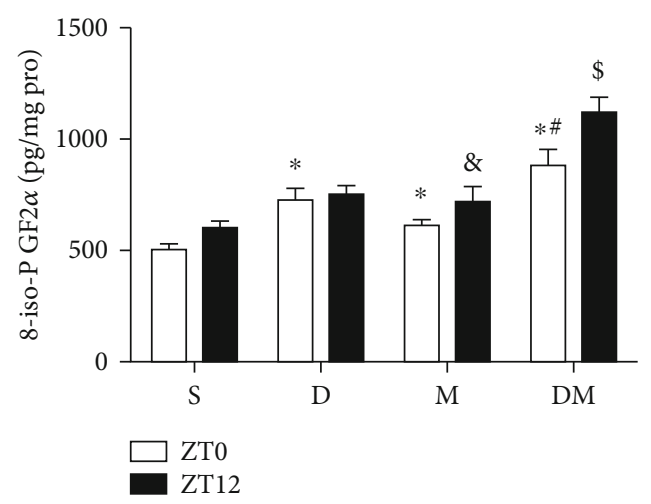

(c)

Figure 4: SOD (a), MDA (b), and 8-iso-prostaglandin-F2 $\alpha$ (c) levels in renal tissues. $n=8$ per group; ${ }^{*} P<0.05$ compared to the values of the $\mathrm{S}$ group; ${ }^{\#} P<0.05$ compared to the values of the M ZT0 group; ${ }^{\$} P$ and ${ }^{\circledR} P<0.05$ compared to the values of the M ZT0 and DM ZT0 groups, respectively.

gene expressions were induced in the $\mathrm{M}$ group while those were inhibited in the D group; however, PER2 gene expression was markedly repressed in the $M$ group while it was induced in the D group. In addition, Nrf2 protein expression was induced in the renal tissues of the $\mathrm{D}$ and $\mathrm{M}$ groups. The PER2 and Nrf2 protein levels were increased in the DM group compared with the $\mathrm{M}$ group, but the CLOCK protein level was decreased in the DM group compared with the $\mathrm{M}$ group. Furthermore, CLOCK, BMAL1, and Nrf2 protein levels were decreased at ZT12 than those at ZT0 in the DM and $M$ groups. In contrast, the PER2 protein level was increased at ZT12 than those at ZT0 in the DM and M groups.

\section{Discussion}

Cardiac surgery-associated AKI is a serious and frequent complication with poor prognosis in clinical practice, which increases the Intensive Care Unit (ICU) length of stay and associated medical cost and affects the long-term survival of patients. The pathogenesis of cardiac surgery-associated AKI is influenced by many factors, such as an exogenous or endogenous toxin, ischemia/reperfusion, inflammation, and oxidative stress [18]. Diabetes is an independent risk factor for both cardiovascular diseases and AKI [3]. Our previous studies have shown that the incidence and severity of MIR and AKI in diabetic patients are significantly higher than those in nondiabetic patients $[15,19]$. In the present study, the kidneys of diabetic rats after MIR showed characteristic histological changes in the renal tubule, including tubular epithelial edema and swelling, lumen dilation, epithelial simplification, nuclear necrosis, cytoplasmic translucency, and vacuolation. Also, the histopathologic grading scores representing renal tubular injury and Scr and BUN levels were increased significantly. As a biomarker for the early diagnosis of AKI, NGAL can accurately and effectively predict AKI related to cardiac surgery, especially in patients with normal baseline renal function $[20,21]$. In the present study, the increase in the NAGL level has been more pronounced in diabetic rats after MIR, which indicates that myocardial injury can lead to adverse renal damage. Taken together, these results indicate that the rat model of AKI following MIR has been successfully established with exacerbation of AKI in diabetic rats.

There are numerous postulated mechanisms describing the reduced synergy between the renal and cardiac functions in the progression of diabetes. Similarly, though the association between AKI and MIR is complex to define, oxidative stress is considered the most important contributing factor. Our previous study findings have shown that MIR reduced the antioxidant capacity of rat kidney and lead to renal injury, and diabetes further aggravated the process of 

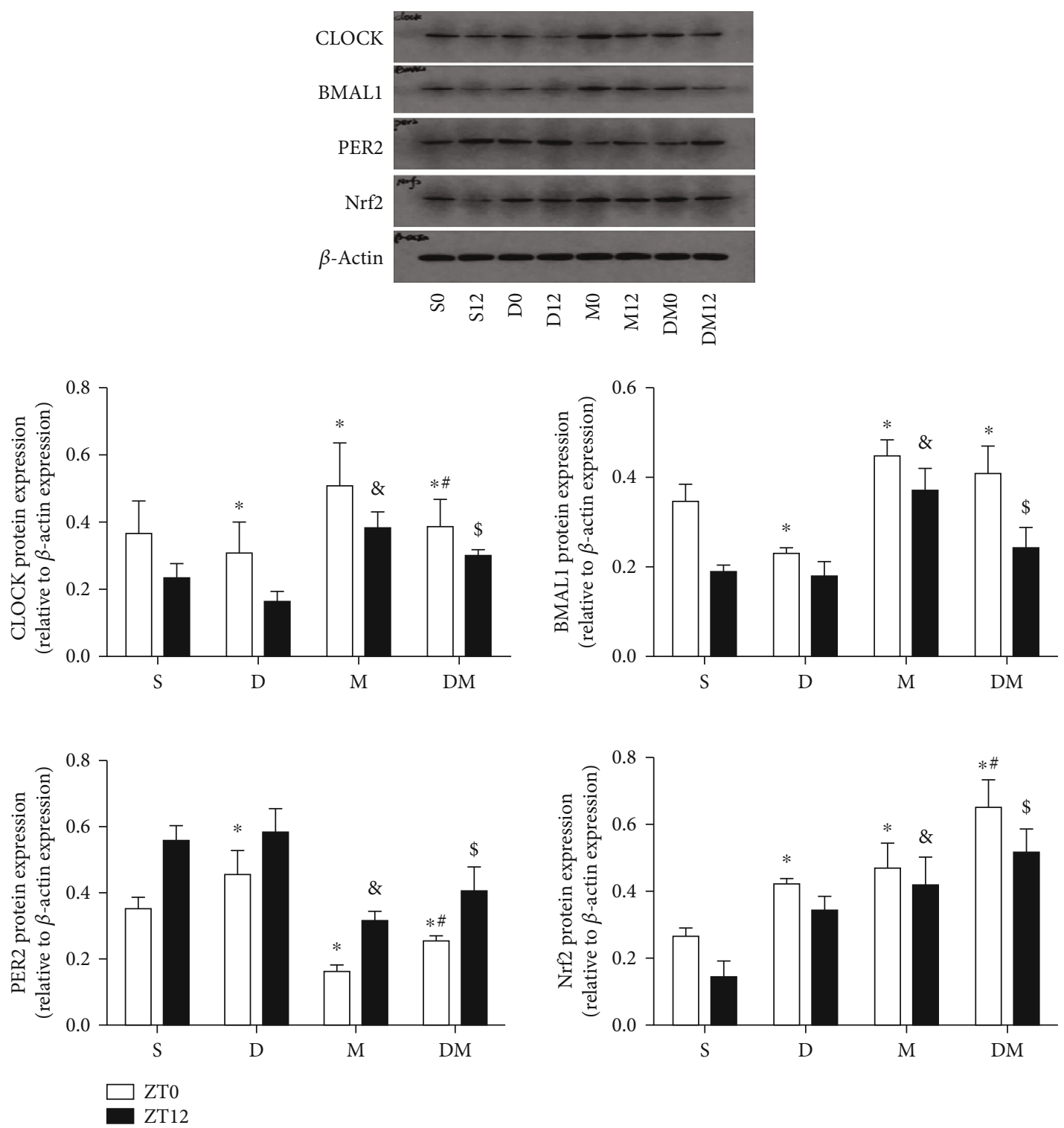

Figure 5: Expressions of CLOCK, BMAL1, PER2, and Nrf2 in the kidney by western blot. $n=8$ per group; ${ }^{*} P<0.05$ compared to the values of $S$ group; ${ }^{\#} P<0.05$ compared to the values of $\mathrm{M} \mathrm{ZT0}$ group; ${ }^{\$} P$ and ${ }^{8} P<0.05$ compared to the values of M ZT0 and DM ZT0 groups, respectively.

oxidative stress in renal injury induced by MIR [9]. Meanwhile, the free radical levels of oxidative stress are the core pathological contributing factor in patients with diabetes, renal, and cardiovascular diseases. Previous studies have shown that, as a marker of lipid peroxidation, the circulating 8 -isopropanol levels are significantly increased in diabetic kidney disease $[22,23]$. The present study results demonstrate that oxidative stress in AKI following MIR is aggravated in diabetic rats as the increase of SOD activity and 8 -iso-PGF $2 \alpha$ and decreased MDA level in the renal tissues. The results also confirmed that oxidative stress in diabetic rats was further aggravated after MIR, which indicates that antioxidant activity has decreased in MIR-induced renal injury with a further decline of antioxidant capacity in diabetic rats.
Circadian rhythm in mammals is related to the periodic oscillation of clock genes, and SCN regulates the expression of 4 important circadian clock genes autonomously, including CLOCK, BMAL1, PER1-3, and CRY1-2 (cryptochromes 1-2). The circadian clock gene BMAL1 is the core promoter of circadian rhythm, which binds to CLOCK to form BMAL1/CLOCK complexes, and then initiates the transcription of PER and CRY genes. Then, there is a negative feedback mechanism where an increase of PER/CRY complexes, in turn, inhibits the activity of BMAL1/CLOCK complexes [4]. Numerous in vivo and in vitro studies have shown that glucose has a significant effect on regulating biological clock signals [24], and hyperglycemia has influenced circadian rhythm on SCN and other specific peripheral tissues in vivo and in vitro $[25,26]$. The STZ-induced diabetic rat's 
myocardium expresses circadian clock genes about 3 hours earlier than those of normal rats on a free diet [27]. In addition, the discordance between cardiac rhythm and SCN rhythm is one of the potential causes of cardiovascular disease in diabetes [28]. In the present study, CLOCK and BMAL1 protein levels are decreased, but the PER2 protein level is increased at night in the DM and M groups. Meanwhile, the renal histologic grading scores, NGAL, and 8-isoPGF $2 \alpha$ levels are significantly higher at night than those in the daytime in the DM and M groups individually, indicating a more severe renal injury at night in the DM and M groups.

Indeed, an increasing number of studies have shown that the circadian clock can regulate cells' protective mechanisms against oxidative stress [11]. Moreover, the latest evidence suggests that under physiological conditions, the body's redox system has a circadian rhythm, and antioxidant genes regulate reactive oxygen species (ROS) rhythmically, which may be the key mechanism for protecting oxidative stress injury in many peripheral organs [12, 29]. Our previous study findings have confirmed that the Nrf2/ARE pathway plays a key role in antioxidant defense to balance oxidative stress induced by ROS in renal IR injury $[9,10]$. Recently, it has been found that Nrf2 is the key gene in the cellar antioxidant defense pathway, and Nrf2 and its downstream antioxidant genes have diurnal variation. The transactivation of Nrf2 is regulated by BMAL1 binding to Nrf2 promoter in the E-BOX region, contributing to the rhythmic activation of the Nrf2/ARE pathway and the rhythmic expression of downstream antioxidant proteins $[12,14,30]$. Subsequently, the rhythmic recruitment and activation of the Nrf2 gene played a critical role in removing ROS to suppress renal IR injury [31]. However, the underlying molecular mechanisms concerning transcriptional control of the circadian clock on the Nrf2 pathway in renal injury induced by MIR and diabetes are still unclear. In addition, the present study results have shown that as similar to CLOCK and BMAL1 protein expressions, Nrf2 protein accumulated in a circadian manner with decreasing levels at night in the DM and M groups. Based on the present study findings, we inferred that the circadian clock genes as endogenous molecular regulators might control the kidney's Nrf2 activation, which exerted protection against the LD variation in susceptibility to MIR and diabetic oxidative injury.

\section{Conclusion}

The present study has shown that the kidney's circadian rhythm may be impaired in diabetic rats, which directly affects the rhythmic aggregation and function of Nrf2 and eventually leads to more severe renal injury of the DM group at night. The results indicated that the AKI following MIR was exacerbated in the diabetes rat at night, through molecular mechanisms involving transcriptional control of the circadian clock on LD activation of Nrf2. Therefore, there is a great challenge to provide an optimal therapeutic strategy based on the circadian clock-associated mechanisms for the treatment of AKI postcardiac surgery in diabetic patients in the near future.

\section{Data Availability}

The data used in the manuscript is provided as Supplementary Materials.

\section{Conflicts of Interest}

The authors declare no conflict of interest.

\section{Authors' Contributions}

Qian Sun and Chong Dong designed the research. Cheng Zeng performed the majority of experiments. Li Du analyzed the data. Qian Sun wrote the paper. Illustrations and proofreading were performed by Chong Dong.

\section{Acknowledgments}

This work was supported by the National Natural Science Foundation of China (No. 82072140).

\section{Supplementary Materials}

Renal score, SCR level, BUN level, NGAL level, SOD level, MDA level, 8-ISO level, CLOCK level, BMAL1 level, PER2 level, NRF2 level, CK-MB level, and LDH level. (Supplementary Materials)

\section{References}

[1] S. T. H. Chew and N. C. Hwang, "Acute kidney injury after cardiac surgery: a narrative review of the literature," Journal of Cardiothoracic and Vascular Anesthesia, vol. 33, no. 4, pp. 1122-1138, 2019.

[2] Z. Q. Kang, J. L. Huo, and X. J. Zhai, "Effects of perioperative tight glycemic control on postoperative outcomes: a metaanalysis," Endocrine Connections, vol. 7, pp. R316-R327, 2018.

[3] D. Montaigne, X. Marechal, T. Modine et al., "Daytime variation of perioperative myocardial injury in cardiac surgery and its prevention by Rev-Erb $\alpha$ antagonism: a single-centre propensity-matched cohort study and a randomised study," The Lancet, vol. 391, no. 10115, pp. 59-69, 2018.

[4] M. Astiz, I. Heyde, and H. Oster, "Mechanisms of communication in the mammalian circadian timing system," International Journal of Molecular Sciences, vol. 20, no. 2, p. 343, 2019.

[5] A. Seifalian and A. Hart, "Circadian rhythms: will it revolutionise the management of diseases?," Journal of Lifestyle Medicine, vol. 9, no. 1, pp. 1-11, 2019.

[6] B. Lebailly, C. Boitard, and U. C. Rogner, "Circadian rhythmrelated genes: implication in autoimmunity and type 1 diabetes," Diabetes, Obesity \& Metabolism, vol. 17, pp. 134-138, 2015.

[7] D. Firsov and O. Bonny, "Circadian rhythms and the kidney," Nature Reviews. Nephrology, vol. 14, no. 10, pp. 626-635, 2018.

[8] S. Yasuda, S. Iwami, K. Tamura et al., "Phase resetting of circadian peripheral clocks using human and rodent diets in mouse models of type 2 diabetes and chronic kidney disease," Chronobiology International, vol. 36, no. 6, pp. 851-869, 2019.

[9] Q. Sun, Z. Y. Shen, W. N. Duan, Q. T. Meng, and Z. Y. Xia, "Mechanism of myocardial ischemia/reperfusion-induced acute kidney injury through DJ-1/Nrf2 pathway in diabetic 
rats," Experimental and Therapeutic Medicine, vol. 14, pp. 4201-4207, 2017.

[10] Z. Y. Shen, Q. Sun, Z. Y. Xia et al., "Overexpression of DJ-1 reduces oxidative stress and attenuates hypoxia/reoxygenation injury in NRK-52E cells exposed to high glucose," International Journal of Molecular Medicine, vol. 38, no. 3, pp. 729736, 2016.

[11] Y. Xia, Z. Cheng, S. Wang, D. Guan, and F. Liu, "Modeling the crosstalk between the circadian clock and ROS in_Neurospora crassa_," Journal of Theoretical Biology, vol. 458, pp. 125-132, 2018.

[12] T. Tamaru, M. Hattori, Y. Ninomiya et al., "ROS stress resets circadian clocks to coordinate pro-survival signals," PLoS One, vol. 8, no. 12, article e82006, 2013.

[13] J. O. Early, D. Menon, C. A. Wyse et al., "Circadian clock protein BMAL1 regulates IL- $1 \beta$ in macrophages via NRF2," Proceedings of the National Academy of Sciences of the United States of America, vol. 115, no. 36, pp. E8460-E8468, 2018.

[14] V. Pekovic-Vaughan, J. Gibbs, H. Yoshitane et al., "The circadian clock regulates rhythmic activation of the NRF2/glutathione-mediated antioxidant defense pathway to modulate pulmonary fibrosis," Genes \& Development, vol. 28, no. 6, pp. 548-560, 2014.

[15] Y. Wu, Z. Y. Xia, B. Zhao et al., “(-)-Epigallocatechin-3-gallate attenuates myocardial injury induced by ischemia/reperfusion in diabetic rats and in $\mathrm{H} 9 \mathrm{c} 2$ cells under hyperglycemic conditions," International Journal of Molecular Medicine, vol. 40, no. 2, pp. 389-399, 2017.

[16] M. E. Erbatur, Ş. C. Sezen, A. C. Bayraktar, M. Arslan, M. Kavutçu, and M. E. Aydın, "Effects of dexmedetomidine on renal tissue after lower limb ischemia reperfusion injury in streptozotocin induced diabetic rats," Libyan Journal of Medicine, vol. 12, no. 1, article 1270021, 2017.

[17] E. Spandou, I. Tsouchnikas, G. Karkavelas et al., "Erythropoietin attenuates renal injury in experimental acute renal failure ischaemic/reperfusion model," Nephrology, Dialysis, Transplantation, vol. 21, no. 2, pp. 330-336, 2006.

[18] Y. Wang and R. Bellomo, "Cardiac surgery-associated acute kidney injury: risk factors, pathophysiology and treatment," Nature Reviews Nephrology, vol. 13, no. 11, pp. 697-711, 2017.

[19] Y. D. Xiao, Y. Y. Huang, H. X. Wang et al., "Thioredoxin-interacting protein mediates NLRP3 inflammasome activation involved in the susceptibility to ischemic acute kidney injury in diabetes," Oxidative Medicine and Cellular Longevity, vol. 2016, Article ID 2386068, 17 pages, 2016.

[20] E. Antonucci, G. Lippi, A. Ticinesi et al., "Neutrophil gelatinase-associated lipocalin (NGAL): a promising biomarker for the early diagnosis of acute kidney injury (AKI)," Acta Bio-Medica, vol. 85, no. 3, pp. 289-294, 2014.

[21] F. Zhou, Q. Luo, L. Wang, and L. Han, "Diagnostic value of neutrophil gelatinase-associated lipocalin for early diagnosis of cardiac surgery-associated acute kidney injury: a meta-analysis," European Journal of Cardio-Thoracic Surgery, vol. 49, no. 3, pp. 746-755, 2016.

[22] R. Clarke, A. Dordevic, S. Tan, L. Ryan, and M. Coughlan, "Dietary Advanced Glycation End Products and Risk Factors for Chronic Disease: A Systematic Review of Randomised Controlled Trials," Nutrients, vol. 8, no. 3, p. 125, 2016.

[23] E. M. Yubero-Serrano, M. Woodward, L. Poretsky, H. Vlassara, G. E. Striker, and AGE-less Study Group, "Effects of sevelamer carbonate on advanced glycation end products and antioxidant/pro-oxidant status in patients with diabetic kidney disease," Clinical Journal of the American Society of Nephrology, vol. 10, no. 5, pp. 759-766, 2015.

[24] M. Toledo, A. Batista-Gonzalez, E. Merheb et al., "Autophagy regulates the liver clock and glucose metabolism by degrading CRY1," Cell Metabolism, vol. 28, no. 2, pp. 268-281.e4, 2018.

[25] T. Nakao, A. Kohsaka, T. Otsuka et al., "Impact of heartspecific disruption of the circadian clock on systemic glucose metabolism in mice," Chronobiology International, vol. 35, no. 4, pp. 499-510, 2018.

[26] L. Qiao, B. Guo, H. Zhang et al., "The clock gene, brain and muscle Arnt-like 1, regulates autophagy in high glucoseinduced cardiomyocyte injury," Oncotarget, vol. 8, no. 46, pp. 80612-80624, 2017.

[27] M. E. Young, C. R. Wilson, P. Razeghi, P. H. Guthrie, and H. Taegtmeyer, "Alterations of the circadian clock in the heart by streptozotocin-induced diabetes," Journal of Molecular and Cellular Cardiology, vol. 34, no. 2, pp. 223-231, 2002.

[28] D. Šoltésová, J. Monošíková, L. Koyšová, A. Veselá, B. Mravec, and I. Herichová, "Effect of streptozotocin-induced diabetes on clock gene expression in tissues inside and outside the blood-brain barrier in rat," Experimental and Clinical Endocrinology \& Diabetes, vol. 121, no. 8, pp. 466-474, 2013.

[29] I. Méndez, O. Vázquez-Martínez, R. Hernández-Muñoz, H. Valente-Godínez, and M. Díaz-Muñoz, "Redox regulation and pro-oxidant reactions in the physiology of circadian systems," Biochimie, vol. 124, pp. 178-186, 2016.

[30] T. Tamaru and M. Ikeda, "Circadian adaptation to cell injury stresses: a crucial interplay of BMAL1 and HSF1," The Journal of Physiological Sciences, vol. 66, no. 4, pp. 303-306, 2016.

[31] A. Desvergne, N. Ugarte, S. Radjei, M. Gareil, I. Petropoulos, and B. Friguet, "Circadian modulation of proteasome activity and accumulation of oxidized protein in human embryonic kidney HEK 293 cells and primary dermal fibroblasts," Free Radical Biology \& Medicine, vol. 94, pp. 195-207, 2016. 